\title{
Itinerarios del Debate Inclusión/Exclusión Respecto a los Dispositivos Tecnológicos. Notas para un Debate Alterno
}

\author{
Itineraries of the Inclusion/Exclusion Debate Regarding \\ Technological Devices. Notes for an Alternate Debate
}

\author{
Armando Ulises Cerón * \\ Eva M. Galán Mireles
}

Universidad Autónoma del Estado de Hidalgo

\begin{abstract}
La noción de exclusión se ha convertido en un tema relevante de las agendas institucionales de los organismos internacionales con el fin de promover condiciones justas erradicándola o al menos controlándola (UNESCO, 2009). Presentamos una serie de reflexiones críticas en las que vinculamos la noción de "exclusión” con el uso de dispositivos tecnológicos y las percepciones sobre éstos a partir de un instrumento aplicado a estudiantes de bachillerato en modalidad virtual en México. Consideramos que la noción de exclusión y su respectivo debate actual, está sobredimensionada y sobrefocalizada en aspectos como los económicos o los etarios; pero estos debates relegan otras dimensiones como las conceptuales y las informacionales olvidando que no solo los cuerpos biológicos envejecen sino también los dispositivos tecnológicos y la información con la que se les opera. Para ello introducimos el debate exclusión / inclusión en las prácticas sociales de forma analítica, y lo contextualizamos en la relación de la técnica y la tecnología dentro de la tipología de los tres tipos de organización social que propone el sociólogo Ronald Inglehart a partir de su proyecto de la Encuesta Mundial de Valores (World Values Survey), para anclar los supuestos desarrollados en los resultados de nuestra encuesta para finalmente arribar a algunas conclusiones preliminares.
\end{abstract}

Descriptores: Exclusión social, Tecnología, Percepción, Aprendizaje en línea, Educación de adultos.

\begin{abstract}
The notion of exclusion has become a relevant issue in the institutional agendas of international organizations in order to promote fair conditions by eradicating it or at least controlling it (UNESCO, 2009). We present a series of critical reflections in which we link the notion of "exclusion" with the use of technological devices and the perceptions about it from an instrument applied to students of baccalaureate in virtual mode in Mexico. We consider that the notion of exclusion and its current debate are oversized and overfocused on aspects such as economic or age; but these debates relegate other dimension such as conceptual and informational forgetting that not only the biological bodies age but also the technological devices and the information which they are operated In order to do so, we introduce the exclusion / inclusion debate in social practices in an analytical way, and contextualize the relationship of technics and technology within the typology of the three types of social organization proposed by the sociologist Ronald Inglehart from his project The World Values Survey, to anchor the assumptions developed in the results of the our survey to finally arrive at some preliminary conclusions.
\end{abstract}

Keywords: Social exclusion, Technology, Perception, E- learning, Adult education.

*Contacto: aceron@uaeh.edu.mx

ISSN: 0718-7378

www.rinace.net/rlei/
Recibido: 30/06/2017

$1^{\text {a }}$ Evaluación: 02/09/2017

Aceptado: 29/09/2017 


\title{
Introducción. La exclusión como práctica social
}

¿Es posible pensar la realidad actual de forma alternativa con algún grado de éxito? Estamos convencidos de que la ciencia social practicada críticamente lo permite. En este sentido, y en concordancia con Berger (1996) es que se propone traer a consideración el debate inclusión/exclusión, relativizando la sobre atención a la que se le ha expuesto, a fin de darle un tratamiento alternativo al oficialmente impuesto por esos organismos internacionales con sus recomendaciones que cuajan en políticas sociales muy concretas, razón por la cual los agentes sociales tienden a asumir de forma ingenua y como importante, problemas que le son impuestos a la sociedad como tales sin llegar a tener plena conciencia de ello. Si bien no se abordarán las definiciones oficiales ni las recomendaciones sobre ellas hechas, sí se abordarán sus efectos en las percepciones de los agentes. $\mathrm{Al}$ respecto,

\begin{abstract}
[...] uno se puede sorprender de que la reflexión sobre las relaciones entre lo normal y lo patológico conceda tan poco espacio al efecto propio del derecho: el derecho, instrumento de normalización por excelencia, en cuanto discurso intrínsecamente poderoso y dotado de los medios físicos para hacerse respetar, está en disposición de pasar, con el tiempo, del estado de ortodoxia, creencia explícitamente enunciada del deber ser, al estado de doxa, de adhesión inmediata a lo que se presupone, a lo normal, como culminación de la norma que queda abolida en cuanto a tal en su perfección. (Bourdieu y Teubner, 2000, pp. 213-214)
\end{abstract}

Por eso, es muy probable que con la misma naturalidad con la que se ha adoptado el uso de la tecnología a nuestra vida cotidiana no recordemos cuándo fue la primera vez que hicimos uso de ella, o que no recordemos cuál fue el primer aparato tecnológico que utilizamos. Si bien se considera a la cultura como la huella del paso del hombre sobre la naturaleza, la sociedad misma y sus dinámicas se convierten en una segunda naturaleza que tiende a ver y a asumir como natural lo que en principio no lo es. En conclusión, el uso de la tecnología también se ha naturalizado (Habermas, 1996).

Esta naturalización de la vida no natural conlleva efectos de los que no siempre se suele ser del todo consciente, uno de los cuales se vincula con la exclusión. Las mismas prácticas mnémicas que nos llevan a recordar algo con preferencia a lo olvidado son manifestación de prácticas excluyentes. Esto nos lleva a preguntar ¿qué es la exclusión?

[...] la inclusión / exclusión, más que un debate actual, es una condición humana.
así como durante el movimiento ilustrado del Siglo XVIII la noción de "prejuicio"
adquirió el sentido negativo que hoy se le da en la ciencia moderna (Gadamer, 1998),
la idea de "exclusión" se ha desbalanceado focalizando fundamentalmente el lado
negativo (no porque sea algo positivo). ¿ No será la "inclusión" una utopía, una
aspiración social más que produce el mundo intelectual? La asimetría social es
también condición de la existencia en sociedad, lo que favorece prácticas excluyentes
que no necesariamente deberían ser consideradas como negativas. El dilema inclusión
/ exclusión no es sino la complicidad ontológica entre las distintas posiciones ocupadas
en los campos sociales. (Cerón, 2016, p. 493)

Sirva lo anterior para introducir nuestra perspectiva sobre la noción de exclusión, tan en boga en tiempos recientes. En un juego de doble dirección, en muchas ocasiones se adoptan términos originalmente científicos para inferir explicaciones sobre las prácticas inmediatas, pero sin la rigurosidad científica original (por ejemplo, cuando las maestras de nivel preescolar, ante la poca o nula efectividad de obediencia del pequeño alumno, le etiquetaban como "hiperactivo", condenándole a otro tipo de trato educativo con esta práctica enclasante, excluyéndole de la educación regular y confinándole a una especial, 
por la falta de pericia en los "modos suaves" y efectivos de dominación por parte de la docente, en palabras de Bourdieu) y, por el contrario, de manera ingenua los científicos toman temas y "problemas" sociales y los asumen como científicos cuando ni en esencia ni en origen lo son. Nos parece que este es el caso del dilema inclusión / exclusión (Bourdieu y Wacquant, 2008).

Una adecuada vigilancia epistemológica sobre el tratamiento del objeto de estudio, que es un principio inherente de una sociología crítica, puede salvaguardar al analista y, sobre todo, al científico social que pretenda ejercer su oficio con la seriedad y la rigurosidad que éste demanda.

Desafortunadamente la sociología empírica sobre el ocio, sobre el trabajo, sobre las ciudades, está hecha por gente humanamente perfecta, pero si puedo decirlo, demasiado humana... La ruptura se opera también contra todo eso. No se hace sociología para tener el placer de sufrir con los que sufren. Habría que tener el coraje de decir no a todo eso. (Bourdieu, 2005, p. 23)

Tenemos la convicción que las prácticas excluyentes sólo han sido focalizadas en su dimensión negativa, sobredimensionándola innecesariamente. Desde la más temprana edad a las crías humanas se les inculca la primera palabra con sentido, un "no" enérgico que limita ciertas acciones y favorece la práctica de otras (Berger y Luckmann, 2003). Por ejemplo, no comer con la boca abierta, no correr en casa ajena cuando se está de visita, evitar comer golosinas que mermen o quiten el apetito, no comer con las manos sin lavar, tener control de la micción o defecar en público, y una gran lista que podría adicionarse a estas acciones socialmente inculcadas que, al practicarlas, demeritan y excluyen a otras. Los beneficios de salud que se obtienen al lavarse las manos en el trato con enfermos en hospitales son mucho más recientes que lo que pudiera pensarse, y enfrentó reticencias sobre las prácticas comúnmente aceptas en su época.

\section{Ignaz Semmelweis, fue un obstetra húngaro que, a mediados del siglo XIX, precediendo los hallazgos de Pasteur y Lister, logró descubrir la naturaleza infecciosa de la fiebre puerperal, logrando controlar su aparición con una simple medida de antisepsia. Debió luchar con la reticencia de sus colegas que no aceptaron sus observaciones que, por primera vez en la historia, fueron apoyadas con datos estadísticos. (Miranda y Navarrete, 2008, p. 1)}

En tanto que fenómeno "reciente", la exclusión como tema de reflexión y debate también incorpora nuevos sujetos y nuevos dilemas que llevan, paradójicamente, a considerar algunos tópicos y a ignorar a otros, excluyéndolos por el simple hecho de no considerarlos bajo los esquemas clasificatorios dominantes. En este sentido, en el apartado metodológico presentamos algunas de las consecuencias de estas prácticas excluyentes.

Este artículo también tiene la intención de poner en la mesa de discusión aquello que consideramos se debate de forma ingenua y poco crítica, y pensamos particularmente en la noción de "exclusión" tal como se ha venido comentando, tomando como referente contextual la relación entre técnica y tecnología en organizaciones sociales recientes y sus modos de apropiación. Tras esta contextualización nos proponemos compartir algunos hallazgos de un instrumento aplicado a estudiantes de bachillerato en modalidad virtual en México que permitan inteligir a la exclusión como práctica social recurrente.

Con un tratamiento metodológico mixto (cuantitativo y cualitativo), y con un enfoque crítico, se rescatan de forma sistemática algunas respuestas que realizaron estudiantes de nivel bachillerato en línea con respecto a las percepciones que tienen sobre su clase social, la tecnología y los usos de ésta en su entorno próximo, dónde se auto incluyen a sí mismos 
y, por ende, dónde se auto excluyen. Para ello se desarrolla un breve contexto de la técnica y la tecnología en el marco de la modernidad reciente en lo que se han llamado las tres revoluciones industriales en relación a tres tipos de sociedades en función de su orientación axiológica y tecnológica, para después focalizar cómo hay percepciones y autopercepciones excluidas y excluyentes de la temática en los sujetos propuestos, y finalmente arribamos a unas conclusiones generales.

\section{Modos de apropiación de la técnica y la tecnología en el contexto sociohistórico}

Derivada de la dinámica de globalización y como consecuencia de la tercera revolución industrial, la tecnología ha sido responsable de muchas transformaciones sociales (Abreu y Medina, 1990). La así llamada Escuela de Frankfurt desde la década de 1920 atisbó los riesgos de la dependencia de la sociedad entera de los beneficios tecnológicos, sobre todo cuando estos últimos están mediados fenoménicamente por intereses económicos. Así como el concepto de tecnología ha cambiado de acuerdo a los contextos en los que se aplica, también se encuentra vinculado con temáticas de inclusión digital y obviamente de exclusión en diversas dimensiones (Torres, 2014).

En este apartado es menester hacer una recapitulación del surgimiento propiamente de la tecnología que permita identificar el sentido de apropiación por parte de la sociedad y de los efectos que ha tenido históricamente. Al respecto es importante recordar que la técnica aparece mucho antes que la ciencia, la cual ha tenido un papel sumamente importante en la evolución humana, por lo que la ciencia es relevante, mayoritariamente para un grupo especializado (Díaz y García-Carmona, 2016). En este sentido, la tecnología se define como técnica científicamente fundamentada, aunque cabe mencionar que es muy posterior el surgimiento de este concepto.

$\mathrm{El}$ género humano, en tanto que especie, posee un conjunto de saberes innatos que se manifiestan en lo que se ha llamado instinto, es decir, el conjunto de tendencias regulares que son propias de la especie. No obstante,

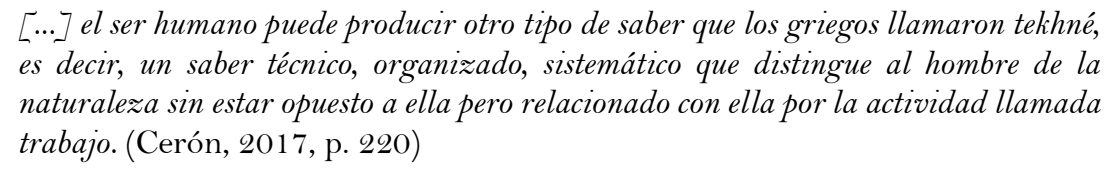

Esta capacidad de producir este saber técnico y aplicarlo al mundo le ha valido al homo sapiens la trascendencia de su original estadio animal al propiamente humano. Con Berger y Luckmann (2003) se puede establecer que el hombre no nace humano, sino que se le forma como humano, y las técnicas que usa para transformar la naturaleza y apropiársela, al usarlas y transmitirlas a las nuevas generaciones le transforman a él de forma simultánea. En este sentido, el ser humano es una esencia técnica en tanto que homo faber y homo oeconomicus. Y a la sistematización de ese saber técnico se le puede definir como tecnología.

Para comprender la relación sociohistórica entre técnica y tecnología, recurrimos al sustento teórico de la Encuesta mundial de valores (World Values Survey, en inglés), proyecto antaño del sociólogo Ronald Inglehart (1994), el cual se basa en la detección de los valores de las sociedades que orientan sus prácticas cotidianas en las diversas esferas sociales. De este modo la investigación detecta tres tipos de organización social muy 
delimitadas: las sociedades premodernas, que están definidas por valores tradicionales, en donde el grupo, la religión, la costumbre y la familia se imponen a los individuos que le componen; su orientación temporal es hacia el pasado como una estrategia de continuidad del grupo, y el honor es un valor fundamental entre sus miembros.

Las sociedades modernas son las que se definen axiológicamente por haber otorgado al Estado y sus instituciones un alto grado de confianza de tal modo que, por ejemplo, la educación formal de sus miembros la arrebata a la familia y se adjudica el rol educativo respaldado por sus sistemas de credencialización escolar (por medio de diplomas, certificados, títulos, etc.). Su orientación temporal es hacia el porvenir, razón por lo que las inversiones y los gastos educativos presentes tienden a ver sus resultados sociales en un futuro próximo.

Por contraste, las sociedades posmodernas son detectables por la orientación hacia los valores individuales; en otras palabras, al individuo se le otorga la autoridad que en los otros tipos de organización tenían la familia, la religión, el Estado y sus instituciones, etc., y ahora estas sociedades buscan la dignificación de sus miembros individuales. Su orientación temporal es hacia el presente, pues se han suplido las necesidades básicas de sus miembros.

Con esto en mente, también es posible detectar los usos y aplicaciones de la técnica y las tecnologías en los quehaceres cotidianos de estas organizaciones sociales (Torres, 2014). En este sentido, las sociedades premodernas son más técnicas que tecnológicas, pues por diversas razones de tipo estructural el uso de las técnicas les ha sido suficientes para la conservación y reproducción tanto individual como grupal, mientras que las sociedades modernas transitan del uso de las diversas técnicas hacia las tecnologías propiamente dichas, y las sociedades posmodernas están totalmente tecnologizadas.

Una vez que se ha tenido en cuenta que la tecnología es una continuidad de la técnica, bajo la mirada de mejorar la calidad de vida, es necesario recordar que la historia de las civilizaciones se vincula estrechamente con la historia de la técnica a través de actividades tan antiguas como la caza, ganadería, la artesanía, medios de transporte, medios de comunicación (la misma escritura) e inclusive las guerras, etc. En todas ellas han jugado un papel importante las máquinas o artefactos que, dicho de otra manera, son los dispositivos tecnológicos como hoy se les nombra de manera más cotidiana (Quintanilla, 2016; Torres, 2014).

Por esta razón, para aprovechar lo anteriormente expuesto se hace necesario abordar el concepto de tecnología, y se rescata la noción de Echeverría (2003), el cual la define como:

\section{[...] un sistema de acciones regladas, industriales y vinculadas a la ciencia, llevadas a cabo por agentes, con ayuda de instrumentos, e intencionalmente orientadas a la transformación de otros sistemas con el fin de conseguir resultados valiosos evitando consecuencias y riesgos desfavorables. (p. 58)}

A partir del siglo xx lo que refiere a la tecnología como una ciencia se ha incrementado de manera estratosférica, dando un enfoque conocido como tecnociencia la cual llega a desempeñar un papel importante en muchas innovaciones tecnológicas (Echeverría, 2003).

Para otros autores, no existe una definición universal de tecnología debido a su uso excesivo en diversos contextos de la vida cotidiana, por lo que se puede entender de diferentes formas a través de los tiempos. Sin embargo, en el sentido más estricto ésta se 
ubica en los siglos XVIII y XIX (Díaz y García-Carmona, 2016) y puede estar constituida bajo los siguientes términos:

- Herramientas, instrumentos, máquinas, artefactos y todo tipo de sistemas.

- Conocimientos técnicos, metodologías, capacidades y destrezas necesarias para poder diseñar y realizar las actividades.

- Recursos humanos y materiales del sistema socio-técnico de producción.

\section{Apropiación tecnológica y exclusión social}

Con una visión objetiva, todas estas variantes del concepto de tecnología pueden ser la causa de la desapropiación del uso dentro de la sociedad y que la filosofía de la tecnología aborda como estudio desde dos vertientes: aquella que se orienta a la comprensión de la naturaleza o la estructura interna de la tecnología y otra que se deriva de los problemas y consecuencias de carácter ético y político (Carvajal, 2012), donde podemos ubicar las problemáticas de exclusión por el carácter humanista.

En este mismo sentido, el determinismo tecnológico de Schumpeter permite identificar los dos lados de la moneda acerca de la tecnología. Para el primer caso, bajo la mirada optimista que señala que los avances tecnológicos pueden llevar a las naciones al desarrollo económico de primer nivel, mientras que el lado obscuro apunta a la promoción de desigualdades sociales y económicas, así como la injusta distribución del trabajo (Montoya, 2004), lo que también lleva implícita la exclusión social.

Para continuar con el determinismo tecnológico, es necesario visualizar los otros factores que los constituyen tales como lo son el etario, el geográfico, el racial, el biológico, el económico o el psicológico que pretenden simplificar la complejidad de los fenómenos sociales y que les atribuyen a los aparatos la facultad de moldear los hábitos y las instituciones.

Si bien es cierto que la tecnología establece la relación práctica entre los conocimientos científicos y las normas de producción, aquélla no sólo determina sino también es determinada por el proceso social que se somete a las reglas de la competencia y el beneficio reproduciendo rasgos de un sistema social capitalista, por lo que resulta complejo entender su impacto directamente sobre las costumbres (Katz, 1998).

Los cambios de percepción respecto a los dispositivos tecnológicos y sus usos pueden variar en consideración con la aplicación en el campo del conocimiento, por su producción en masa, así como la demanda y/o la aceptación a la población a la que va dirigida. Por otra parte, la dinámica de actualización se hace cada vez más rápida, lo que subraya que la tecnología y su apropiación social tienen un papel primordial. Con Dussel (1985), se puede decir que la posesión de un bien, que es la relación objetiva, directa del poseedor con el dispositivo, entra en movimiento dialéctico con la relación intersubjetiva que es la propiedad del mismo, o sea, el reconocimiento de derechos jurídicos del poseedor sobre el dispositivo; la resultante es la apropiación, o sea, el uso objetivo-subjetivo de un dispositivo con derechos jurídicos.

En este sentido, es importante comprender que la posesión material de un dispositivo tecnológico, en tanto que capital cultural objetivado, requiere también de capital económico. En otras palabras, no basta con tener el criterio informacional suficiente para 
elegir un dispositivo: la elección del mismo también depende de la capacidad económica para asegurarse su propiedad jurídica: "Por lo tanto, los bienes culturales pueden ser apropiados o bien materialmente, lo que presupone capital económico, o bien simbólicamente, lo que presupone capital cultural" (Bourdieu, 2000a, p. 144). De este modo, un dispositivo tecnológico, en tanto que bien cultural, suele ser el producto de la intersección de, al menos, dos tipos de capitales, el cultural y el económico.

La acotación anterior se vuelve necesaria para comprender la trayectoria de los modos de apropiación de los bienes culturales, pues éstos, al igual que los agentes sociales, poseen también un itinerario en el espacio social, por lo tanto, una dinámica, una evolución. Este tipo de comprensión acerca de los dispositivos tecnológicos permite anular el efecto del presentismo ingenuo, que lleva a considerar las condiciones actuales de las prácticas como si fuesen únicas y hasta universales (Bourdieu, 2005). De ahí que, por ejemplo, se deriven las reacciones de asombro de jóvenes agentes sociales cuando escuchan que los adultos cercanos a él tuvieron experiencias tan "raras" como el tener que levantarse frente al aparato televisor y girar la perilla del dispositivo in situ si se quería cambiar de canal cuando, por contraste, ellos "siempre" lo han hecho con control remoto, e incluso más recientemente desde su Smartphone, si se tiene descargada la respectiva aplicación que les permita realizarlo (Álvarez y Márquez, 2010).

De esta manera, artefactos tan "simples" como lo pueden ser una puerta y el sistema de bisagras que le sostienen, así como la llave que le permiten cerrarse o abrirse, son dispositivos tecnológicos que hemos asumido de manera cuasi natural por la cotidianidad de su uso, y por ello rara vez son objeto de atención y mucho menos de admiración o de asombro. Artefactos de uso común como la cuchara, el plato, la cama, la estufa de gas, el sistema de ductos de agua y sus perillas en las modernas casas habitación, etc., forman parte ya del panorama habitual como para los animales en estado natural lo puede ser el río, el bosque, la selva, la montaña, etc. Es por esta razón que se ha insistido en que la sociedad se constituye como una segunda naturaleza para la especie humana (Habermas, 1996).

Ahora bien, las técnicas usadas en sociedades premodernas a lo largo de su trayectoria por este mundo son variadas: para conservar alimentos se ha usado el salarlos, congelarlos, ahumarlos, endulzarlos, fermentarlos, esterilizarlos (pasteurización), deshidratarlos, envasarlos en aceite, etc. De manera particular, en las culturas precolombinas en México

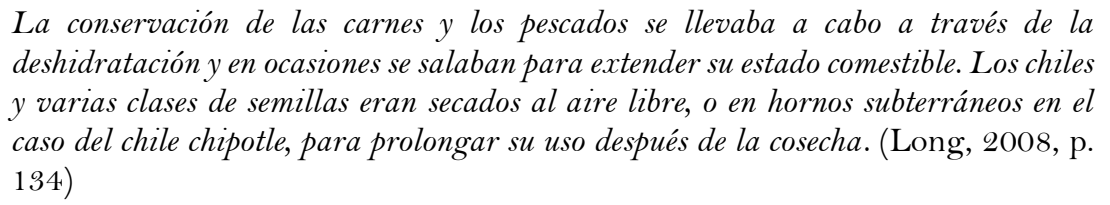

Con estas técnicas, este tipo de sociedades premodernas se han asegurado la producción y la reproducción de su existencia. Con el desarrollo de la ciencia aplicada a la producción fabril en masa, las técnicas devienen en tecnologías muy específicas y, en el caso de las sociedades modernas, a partir del siglo XVIII la técnica de conservación ha derivado en tecnologías de refrigeración, produciendo un artefacto como el refrigerador. La primera patente de un rústico refrigerador moderno data de 1834, otorgada a Jacob Perkins, y en 1879 se creó el primer refrigerador comercial para uso doméstico (Refrigeracionner, s/f). Cabe mencionar que su invención fue producto lateral de otras aplicaciones, sobre todo por científicos; es lo que suele llamarse la serendipia, el descubrimiento inesperado, pero afortunado, cuando se buscaba intencionalmente otra cosa. De este modo, el refrigerador 
se ha vuelto otro artefacto más del panorama cotidiano en las sociedades modernas. Por contraste, las sociedades posmodernas combinan e incorporan tecnologías digitales a los modernos refrigeradores tales como el acceso a internet, el control del dispositivo por medio del Smartphone, etc. Elegir entre una técnica de conservación de alimentos, suele excluir a las otras.

Se puede asegurar que artefactos tecnológicos como la radio, el automóvil o los electrodomésticos llegaron para modificar los hábitos y las costumbres, y en su uso ellos mismos han sido transformados. Siguiendo la misma lógica expositiva anterior, se puede recordar cómo el relato oral es, por excelencia, un importante medio de socialización en sociedades premodernas, y más cuando se practica en relaciones sociales asimétricas donde alrededor de los ancianos venerables de la tribu, los jóvenes van aprendiendo los mitos propios de su grupo y de su entorno (Torres, 2014).

$\mathrm{Si}$ se piensa en las sociedades modernas, propiamente las occidentales del siglo $\mathrm{Xx}$, conviene recordar cómo la radio, y después la televisión fueron dispositivos tecnológicos de entretenimiento, tomando el lugar del relato oral de las organizaciones sociales anteriores, e introduciéndose en la vida privada de los sujetos sociales, propiamente en el contexto familiar. Si se buscan imágenes sobre la historia de la radio en internet, es muy probable encontrar cómo en su momento la radio, y después el televisor, fueron el centro de ocio familiar por excelencia (Álvarez y Márquez, 2010).

Con el desarrollo tecnológico y los intereses de mercado, el precio de los dispositivos mermó y, de ser aparatos usados como centro de atención familiar (sometiendo a todos sus miembros a prestar atención al gusto impuesto por quien solía ejercer el rol dominante, por lo general, los adultos como el padre, la madre, los abuelos, etc.), se disipó este punto de gravitación del ocio familiar con la modalidad de que cada miembro tuviese su propio dispositivo, hasta llegar a los artefactos más personalizados como el teléfono celular o bien la computadora personal (Pacheco, 2008).

\section{El referente empírico y el tratamiento metodológico}

Este trabajo de investigación en su dimensión empírica tiene características metodológicas cuantitativas y cualitativas con un diseño no experimental de carácter diagnóstico, y se aplicó como un instrumento en línea a una muestra intencional no probabilística de 58 estudiantes de un programa de bachillerato bajo la modalidad virtual en México, el cual abrió la oportunidad para detectar una gama diversa de perfiles.

La aplicación de este instrumento se llevó acabo en el primer semestre del 2017. Cabe mencionar que, aunque el principal objetivo de este instrumento fue conocer las percepciones respecto al concepto de tecnología que permitiesen identificar tipos de exclusión tecnológica, también se identificaron otros aspectos que permiten detectar la dimensión social de estas prácticas.

Una de las metas específicas fue el identificar las concepciones posibles respecto a los dispositivos tecnológicos que develen la asunción naturalizada del uso de artefactos tecnológicos. Antes de la aplicación del instrumento teníamos el supuesto de que la población a encuestar tendría una predisposición conceptual de lo que es la tecnología al remitirla prácticamente a dispositivos digitales, lo que apareció de forma consistente en los registros del instrumento aplicado. 
Lo anterior también nos llevó a incorporar ítems que nos permitiesen identificar las percepciones de complejidad de uso de los dispositivos tecnológicos que ellos mismos incorporan a su vida cotidiana. Dicho instrumento constó en tres secciones. La primera correspondió a los datos de identificación personal y condiciones socio-económicas, mientras que en la segunda sección se establecieron los ítems de percepción, uso y exclusión de la tecnología a través de preguntas cerradas y abiertas (en su mayoría) que otorgaran posibilidad de expresión acorde con las condiciones contextuales de los encuestados; finalmente la tercera estuvo dedicada a conocer el interés de continuar participando en próximos ejercicios derivados de este primer acercamiento.

\section{Resultados}

A partir de un análisis de datos recopilados se identificó en primera instancia que la edad promedio de los participantes es de 35 años, sin embargo es importante mencionar que esta variable, que en apariencia no es determinante sino por el contrario, se podría identificar etérea por ser una característica de la misma modalidad virtual, al permitir este servicio de bachillerato el ingreso de estudiantes de diversas edades las cuales oscilan entre 15 a 58 años, en realidad oculta una serie de prácticas excluyentes de forma institucionalizada.

Al respecto, conviene señalar que hay, ciertamente, una edad biológica, pero también existe una edad social, o más bien, socialmente consagrada para realizar cierto tipo de actividades, fuera de las cuales es prácticamente imposible acceder salvo por el coste de romper las normas externas y exponerse a las sanciones correspondientes, dicho esto y de acuerdo con la temática abordada en este trabajo conviene mencionar que también los dispositivos tecnológicos tienen un tiempo de utilidad y una edad socialmente permitida, por ejemplo las unidades de almacenamiento digital que han sido conocidas como diskettes que en su momento fueron de gran utilidad inclusive para instalar sistemas operativos de arranque que hoy se encuentran ya caducados, razón por la que el mercado no los produce más y por ende, ni se distribuyen ni se consumen.

Con relación a la exclusión, las personas que adquirieron las habilidades de uso de esos dispositivos particulares, ahora es una competencia caduca al no poder operar los dispositivos actuales; por lo tanto, quien pretenda operar con los esquemas anteriormente adquiridos tendrá dificultades para operar los nuevos dispositivos digitales y de igual manera los novatos digitales. Esto podría considerarse en términos teóricos como el efecto Quijote, al haber un desfase entre las condiciones actuales y los esquemas antaños que en su momento regularon las prácticas de operación pero que hoy no funcionan más (Bourdieu, 2000b)

Algo similar ocurre con los espacios formales para realizar los estudios básicos: la edad biológica tiende a influir hacia dónde será posicionado el agente social concebido como educando. En este sentido, se puede hablar con propiedad de una edad escolar. Quienes por alguna razón han pasado el límite de la edad institucionalmente permitida, tienden a ubicarse o a ser ubicados en los espacios alternos a los presenciales. La modalidad virtual en línea, al igual que la educación para adultos incorporan a agentes que ya no pueden ser incluidos en la modalidad tradicional concebida para agentes con trayectorias sociales y escolares regulares. Este es un tipo de exclusión institucionalizada y de la que poco se 
cuestiona por asumirse como una práctica legítima. Se puede ver la figura 1 donde se detecta la edad oficial en México para ingresar a estudios igualmente oficiales.

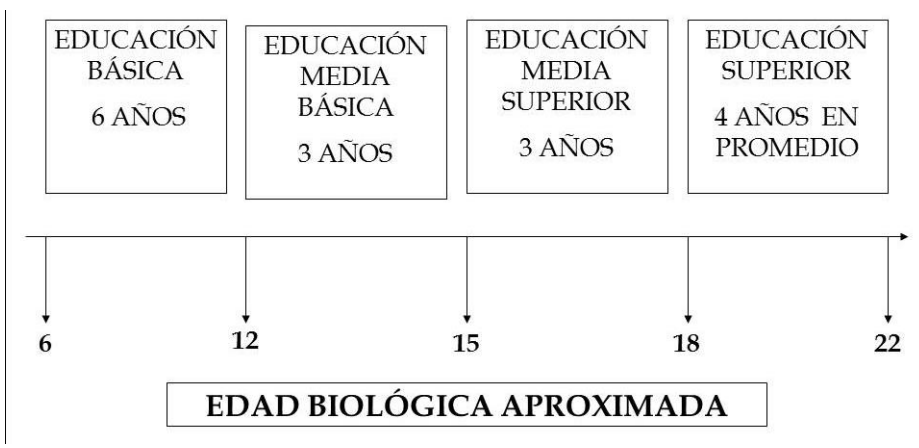

Figura 1. Edad escolar en México

Fuente: Recuperado de Cerón (2007, p. 185).

$\mathrm{Al}$ respecto, Berger (1996) nos permite considerar que la sociedad tiende a considerar que se puede (y se permite) ser hippie, rebelde o revolucionario a los dieciocho años; pero serlo a los cuarenta, ni es normal ni es permitido, salvo al riesgo de ser sancionado por el orden socialmente instituido. De ahí que la edad para estudiar en estos espacios virtuales tienda a ser un signo distintivo que permite su exclusión del sistema regular y posibilita su inclusión en esta modalidad alterna.

En el mismo sentido se encuentra la residencia al encontrar una diversidad en sus respuestas. Se registró que 34 de los 58 sujetos $(58.6 \%)$ radican fuera de las tres entidades más desarrolladas en México: Ciudad de México, Guadalajara y Nuevo León, lo que en este perfil sociológico contribuye a reforzar la idea de que los estudiantes en esta modalidad virtual están excluidos de las entidades con mayor concentración y riqueza de capital cultural, por su solo posicionamiento geográfico. Si bien éste no es un factor determinante para ser excluidos del sistema educativo presencial, es un indicador que permite comprender que la exclusión de las entidades más ricas económica y culturalmente en el territorio nacional contribuye a la adscripción a la modalidad virtual.

Otros factores que influyen en el posicionamiento en esta modalidad educativa y su concomitante exclusión del sistema presencial son el género, y el estado civil. La caracterización de la muestra permite identificar los siguientes aspectos: que el $76 \%(44)$ pertenece al género femenino, mientras que el 24\% (14) son hombres; de los 58 participantes el 48\% (28) son casadas (os), un 33\% corresponde a los solteros (as), el $11 \%$ a la unión libre, el $7 \%$ divorciado (a) y solo el 1\% separada. Que tres cuartas partes de la muestra sean mujeres y gran parte de ellas en estado de compromiso familiar, permite visualizar cómo las sanciones sociales (si así se le entendiese a la modalidad virtual respecto a la presencial) llevan una carga de género y condición civil. En una segunda fase de esta investigación se aplicarán entrevistas para confirmar o refutar este aspecto.

En tanto que instrumento racionalmente pensado en detectar percepciones, se solicitó a los encuestados que hiciesen un autoanálisis de en qué clase social se miran. De acuerdo a Pierre Bourdieu (1998), el habitus, estructura externa internalizada, posee la característica de la indeterminación toda vez que, al estar ubicado en el espacio social, siempre encontrará agentes sociales que estén por encima de ellos por poseer más recursos que los actualmente poseídos, pero a la vez identificará que otros más poseen menos de los que él 
tiene y agencia. En este sentido, el habitus de los agentes sociales tiende a colocarlos en una especie de gran clase media. Este supuesto se operó desde el instrumento aplicado.

Esta ofuscación es particularmente visible en las clases medias y, en especial, en las nuevas fracciones de estas clases que, en calidad de lugares de indeterminación situados en posición inestable en la estructura social, personifican en su más alto grado la propiedad, característica de la clase en su conjunto, de hacer coexistir individuos y trayectorias extremadamente dispersas. (Bourdieu, 1998, p. 110)

De la muestra de participantes, 43 de los 58 mencionaron pertenecer a la clase social "media" lo que representa el $74 \%$, el resto se considera entre media baja y baja. Cabe mencionar que esta pregunta se aplicó de manera abierta por lo que su respuesta se observa subjetiva de acuerdo a la propia percepción, y conforme a la acotación conceptual mencionada. Evidentemente, al autoincluirse como "clase media", los encuestados se autoexcluyen de las otras posibilidades.

Respecto a la pregunta de mencionar cinco dispositivos tecnológicos que utilicen con mayor frecuencia, donde el primero corresponde al más frecuente y el último al menos frecuente, se distinguió que el $56 \%$ mencionó que es el celular el dispositivo electrónico que más usan y un $25 \%$ respondió que es la computadora, aunque se identifica también que el $100 \%$ de la muestra refirió ambos como posibles respuestas. Cabe mencionar que nuestra postura respecto a los dispositivos tecnológicos en esta pregunta tenía la intención de abarcar a la vez a los dispositivos técnicos.

En relación a lo anterior, vale la pena mencionar lo que Pierre Bourdieu define como alodoxia, es decir, el error de percepción y, sobre todo, de acción de los agentes sociales respecto a las definiciones dominantes.

La lucha que se ventila en el conocimiento del mundo social no tendría objeto si cada
agente encontrara en sí mismo el principio de un conocimiento infalible de la verdad
de su condición y de su posición en el espacio social y si los mismos agentes no pudieran
reconocerse en discursos y clasificaciones diferentes (según la clase, la etnia, la religión,
el sexo, etc.) o en valoraciones opuestas de los productos de los mismos principios de
clasificación; pero los efectos de esta lucha resultarían totalmente imprevisibles si no
hubiera ningún límite a la alodoxia, al error de percepción y sobre todo de expresión,
y si la propensión a reconocerse en los diferentes discursos y en las diferentes
clasificaciones propuestas fuera igual para todos los agentes, cualquiera que sea su
posición en el espacio social-por tanto, sus disposiciones-y la estructura de ese espacio,
la forma de las distribuciones y la naturaleza de las divisiones según las cuales se
organiza realmente tal espacio. (Bourdieu, 2001, pp. 100-101)

Lo anterior es un tópico que no es de menor valía respecto a los otros hallazgos presentados. En efecto, ante la solicitud de que los encuestados referencien los cinco dispositivos tecnológicos de mayor uso por ellos, nótese que por tales sólo se mencionaron dispositivos digitales.

Algo similar ocurre con el siguiente ítem, que refuerza lo anteriormente planteado. En el caso de la pregunta para conocer el rango de edad en el que consideraban haber comenzado a utilizar dispositivos tecnológicos, se otorgaron las siguientes posibilidades que delimitan los periodos escolares de alguna manera: a) entre mi nacimiento y los 5 años, b) entre los 6 años y los 11 años, c) entre los 12 y los 14 años, y d) después de los 15 años. Se identificó que el $62 \%$ afirma la opción "d", un $24 \%$ la opción "c" y $9 \%$ corresponde a la letra "b" y el $5 \%$ restante a la opción "a”. Lo anterior refuerza la idea de la alodoxia sugerida toda vez que los dispositivos tecnológicos están a nuestro alcance desde nuestro nacimiento (la casa 
habitación ocupada; la cuna o la cama para dormir; los utensilios para cocinar y para comer, etc.).

Ante la pregunta sobre cuál es el dispositivo que más se les facilitó aprender a usar, el 47\% contestó que es el celular; mientras que un $29 \%$ señaló que es la computadora y el resto se encuentra distribuido entre la laptop, la televisión y otros.

En contraste el dispositivo que más se les dificultó aprender es la computadora con un $41 \%$; pero llama la atención que el $19 \%$ mencionara que ningún dispositivo tecnológico es difícil de aprender, en tercer lugar, se encuentra el celular con un $12 \%$, la tablet con un $10 \%$, laptop $5 \%$ y el resto encuentra otros dispositivos, por ejemplo, la impresora, o la Smart TV.

\section{Discusión y conclusiones}

Conscientes de la limitación del espacio otorgado, en esta parte recuperamos algunas conclusiones de lo abordado a fin de que contribuyan a considerar alternativas pedagógicas a las oficialmente reconocidas, al menos en cuanto a la concepción dominante del debate inclusión / exclusión.

En primer lugar, se puede aseverar que la exclusión es una práctica cotidiana de los agentes sociales, y no siempre debería ser considerada negativa o que produce efectos negativos. Este breve itinerario propuesto ha permitido argumentar que la exclusión / inclusión, más que un debate de actualidad, ejemplifica modos de hacer en la vida diaria más elemental. Elegir una plataforma como Windows, Mac, Linux., etc. implica elegir y priorizar ciertas prácticas informáticas en detrimento de otras. Como este ejemplo, se multiplican muchos otros que evidencian a la exclusión como una práctica social. Es verdad que toda afirmación debe relativizarse para no llevarse al extremo del dogmatismo. Baste con decir que hay grados y tipos de exclusión que se han mencionado aquí, pero al no ser el objeto de este artículo, sólo se presentan de forma implícita.

¿En qué radica el error de percepción que a la vez lleva a una especie de exclusión conceptual? En que los esquemas cognitivos de los encuestados sólo pueden pensar como "dispositivo tecnológico" a los dispositivos digitales (celular y computadora, básicamente), lo cual les lleva a una exclusión práctica de otros artefactos tecnológicos tales como la estufa, el automóvil, la puerta, etc. En este sentido, las definiciones simbólicas dominantes sobre lo que sea un "dispositivo tecnológico" tiende a limitar el alcance de los esquemas cognitivos sólo hacia los digitales.

Otro aspecto más que se ha detectado es que la exclusión también tiene su propia dimensión conceptual. El instrumento aplicado permitió detectar que los encuestados tendieron a reconocer como "dispositivos tecnológicos" propiamente a los dispositivos digitales, excluyendo con ello a los otros artefactos tan tecnológicos como lo son la estufa, la puerta, la regadera, etc.

Las prácticas de inclusión/exclusión también operan de manera institucionalizada. En efecto, si se pone atención a los perfiles de los estudiantes de modalidad virtual, sólo el $33 \%$ de ellos son solteros, lo que lleva a detectar que la gente con compromisos formales son los que se vuelven ubicables para esta modalidad educativa, y que por esa condición además de la etaria (la media de edad es de 35 años), tienden a ser excluidos de la modalidad presencial. 
El tránsito de un tipo de sociedad a otra (premoderna, moderna y posmoderna), si bien no es secuencial, por la manera de apropiarse de las técnicas y las tecnologías a su alcance, se autodefinen en prácticas muy particulares. Por ejemplo, las sociedades premodernas giran en torno a los relatos de los ancianos de las tribus, convirtiendo a esos cuerpos biológicos como dispositivos de transmisión oral. Por contraste, las sociedades modernas, al tener dispositivos como la radio o el televisor como centro de entretenimiento familiar, han desplazado los relatos tribales por los de los anunciantes comerciales. Este tipo de sociedades también transitaron de las prácticas grupales a las individuales cuando los dispositivos fueron siendo apropiados en los cuartos privados de los miembros de las familias, donde podían ser artefactos que todavía podían intercambiarse. Las sociedades posmodernas se caracterizan por el uso de dispositivos cada vez más personalizados y menos intercambiables, toda vez que se les ha ligado a la identidad personal de sus poseedores.

En este sentido, es posible considerar que no sólo los cuerpos biológicos caducan al envejecer, sino también caducan los conocimientos de ellos respecto al mundo y sus entidades. El instrumento aplicado permitió detectar, por ejemplo, que ante la pregunta de cuál era el dispositivo tecnológico que más trabajo les había costado aprender, un sujeto de edad "adulta” mencionó a la Smart TV. En efecto, el conocimiento que en sus condiciones originales le llevó a aprender cómo usar un televisor (estando conectado a la corriente eléctrica, con una antena para captar la señal, girar la perilla selectora de canal y ajustar el volumen de audio era información suficiente para apropiarse de él), ahora frente a la Smart TV ese conocimiento es insuficiente y, por lo tanto, ha caducado por no actualizarse.

De forma acrítica suelen tomarse las recomendaciones impuestas por quienes tienen el poder y la legalidad para hacerlo. Una de esas recomendaciones que vienen de la UNESCO gira en torno a la "alfabetización digital", y se le toma como una panacea para las sociedades. Pero por sobredimensionar los posibles beneficios, se excluyen los riesgos posibles. Y para no reiterar los más conocidos (robo de identidad, plagio, la no siempre originalidad de lo que se encuentra en internet, etc.), podría pensarse a la vez en un necesario proceso de "desalfabetización digital", sugerido por el uso indiscriminado y poco reflexivo de, por ejemplo, herramientas de corrección ortográfica en procesadores de texto de forma automática, tanto en computadoras como en teléfonos celulares, pues suele ocurrir que se aceptan sin más las "sugerencias" de escritura. Términos como "pasó" y "paso", al ser ortográficamente correctas, no son señaladas como erróneos por dichos procesadores de texto. Quien escribe, se supondría que debería conocer al menos las reglas elementales de la ortografía. Pero al aceptar las "sugerencias" de escritura y excluir lo que el procesador no detecta como error, se filtran errores básicos como la falta de distinción entre un "tú" y "tu”; “amo" y "amó"; "él” y "el”, etc. Lo mismo ocurre con la inserción automatizada de referencias y citas en textos académicos.

Que el debate inclusión/exclusión sea un tema de rigor en las agendas actuales de los organismos internacionales, no debería significar que debería asumirse con la misma importancia que ellas le otorgan, ni mucho menos por quienes deberían pretenden hacer ciencia social rigurosa. Queda siempre la posibilidad de jugar el juego serio de la ciencia de forma no seria.

Por ello es importante recordar que la educación crítica no sólo puede ayudar a entender de manera alterna el uso de la información dentro del proceso de enseñanza y aprendizaje sino que puede ser más crítica y reflexiva sobre el determinismo tecnológico, para que éste 
de verdad pueda aportar a la construcción de una sociedad más equitativa y de sujetos reflexivos (García, 2014).

El sociólogo reflexivo (en el que se pueden incluir todos aquellos que toman como objeto de reflexión a la sociedad, y no sólo porque se ha formado así de forma disciplinar) tiene tres opciones ante las imposiciones conceptuales y las definiciones legítimas: someterse acríticamente a ellas y asumirlas tal como son y, a partir de ellas definir el mundo que pretende explicar, o bien trascender este nivel y "dialogar" con esas definiciones en una pretendida relación de pares, homóloga, pero conllevando aún el riesgo de asumir como importantes las temáticas que les sugieren las "recomendaciones", sin que necesariamente lo sean, ocultando en ese reconocimiento el desconocimiento de los modos sutiles de imposición y dominación de los que las sugieren. Este trabajo no ha pretendido enfatizar la necesidad de alfabetización digital, por el contrario, abrir una brecha a lo que consideramos una "desalfabetización digital" derivada del abuso de las herramientas digitales, por ejemplo, del corrector automático de textos, el corrector ortográfico, la inserción de citas, herramienta copiar y pegar, entre otras.

En este sentido, el reto para una sociología crítica es trascender la trascendencia: ni aceptar acríticamente las imposiciones temáticas ni asumirlas sin más para un debate también impuesto. La perspectiva crítica, en vez de someterse, somete las definiciones y las temáticas impuestas para ser conscientes de esas sutiles formas de dominación y, al reconocerlas, forjar la posibilidad para detectarlas y enfrentarlas con la dignidad que da el campo científico. De no practicar la ciencia social de este modo, se corre el riesgo de sufrir con los que sufren sus miserias y sus limitaciones, en vez de proveerles herramientas analíticas que posibiliten que el dominado sea consciente de la dominación en la que le han posicionado los que tienen las posiciones dominantes, y con ese conocimiento neutralizar en alguna medida los efectos de su dominación. Respecto a los que piensan a la exclusión como un grave riesgo y, por ende, suelen sugerir la inclusión de los excluidos, están en la condición de tratar el asunto con un buen corazón, y hasta con nobles sentimientos. Pero, como advierte Pierre Bourdieu (2005, p. 22), "con buenos sentimientos se hace la mala sociología”.

\section{Referencias}

Abreu, L. y Medina, S. (1990). La tercera revolución industrial y las nuevas exigencias del posgrado. OMNIA, 6(18), 7-10.

Álvarez, G. M. y Márquez, E. (2010). La relación actual entre la familia y la televisión como medio de comunicación influenciador o socializador (Tesis de especialización no publicada). Universidad de la Sabana, Instituto de la Familia, Bogotá, Colombia.

Berger, P. (1996). Una invitación a la sociología. Ciudad de México: Limusa.

Berger, P. y Luckmann, T. (2003). La construcción social de la realidad. Buenos Aires: Amorrortu.

Bourdieu, P. (1998). La distinción. Criterio y bases sociales del gusto. Madrid: Taurus.

Bourdieu, P. (2000a). Poder, derecho y clases sociales. Barcelona: Desclée de Brower.

Bourdieu, P. (200b). Cosas dichas. Madrid: Gedisa.

Bourdieu, P. (2001). ¿Qué significa hablar? Barcelona: Akal.

Bourdieu, P. (2005). Capital cultural, escuela y espacio social. Ciudad de México: Siglo XXI. 
Bourdieu, P. y Teubner, G. (2000). La fuerza del derecho. La Paz: Siglo del hombre editores.

Bourdieu, P. y Wacquant, L. (2008). Una invitación a la sociología reflexiva. Buenos Aires: Siglo XXI editores.

Carvajal, A. (2012). La filosofía de la tecnología como disciplina. Revista de Ciencias Sociales y Humanidades, 4, 16-20.

Cerón, A. U. (2007). Producción y reproducción de posicionamientos sociales inciertos. Habitus, elección e indeterminación en los estudiantes de la carrera de sociología de la FES Aragón, UNAM (Tesis doctoral no publicada). Universidad Nacional Autónoma de México.

Cerón, A. U. (2016). La razón margi-nada. Notas al margen de una exclusión institucionalizada. Eikasia. Revista de Filosofía, $71(1)$, 493-509.

Cerón, A. U. (2017). Sobre la (in)utilidad de la razón filosófica en el contexto de la razón educativa. Eikasia. Revista de Filosofía, 73(1), 217-235.

Díaz, J. y García-Carmona. (2016). Una controversia de la historia de la tecnología para aprender sobre naturaleza de la tecnología: Tesla vs. Edison. La guerra de las corrientes. Enseñanza de las Ciencias: Revista de Investigación y Experiencias, 1(34), 193-209.

Dussel, E. (1985). La producción teórica de Marx. Un comentario a los Grundrisse. Ciudad de México: Siglo XXI editores.

Echeverría, J. (2003). La Revolución tecnocientífica. Barcelona: FCE.

Habermas, J. (1996). Ciencia y técnica como ideología. Ciudad de México: REI.

Inglehart, R. (1994). Modernización y posmodernización: La transformación de la relación entre desarrollo económico y cambio cultural y político. Recuperado de http:/ / estepais.com/site/?cat=12\&n=38

Katz, C. (1998). Determinismo tecnológico y determinismo histórico-social. REDES, 5(11), 37-51.

Long, J. (2008). Tecnología alimentaria prehispánica. Estudios de cultura náhuatl, 39, 127-136. h

Miranda, M. y Navarrete, L. (2008). Semmelweis y su aporte científico a la medicina: Un lavado de manos salva vidas. Revista Chilena de Infectología, 25(1), 54-57. https://doi.org/10.4067/S0716-10182008000100011

Montoya, O. (2004). Schumpeter, innovación y determinismo tecnológico. Scientia et Technica, $10(25), 209-213$.

Pacheco, F. L. (2008). Análisis Cuantitativo de la Programación de XHCOM 105.9 (Tesis de grado). Universidad de las Américas.

Torres, A. J. (2014). Los dispositivos tecnológicos cotidianos, como objetos de enseñanza. Estudio de la problemática inherente a su elaboración como materiales de aula. Granada: Universidad de Granada

Quintanilla, M. (2016). Tecnología: Un enfoque filosófico y otros ensayos de filosofía de la tecnología. Ciudad de México: FCE.

Refrigeracionner, (s/f). Historia de los refrigeradores domésticos. Recuperado de http://refrigeracionner.com/historia-de-los-refrigeradores-domesticos/

UNESCO. (2009). Directrices sobre politicas de inclusión en la educación. París: ONU. 


\section{Breve CV de los autores}

\section{Armando Ulises Cerón}

Es Licenciado en Sociología por la Universidad Nacional Autónoma de México, Maestro en Filosofía de la Ciencia por la Universidad Autónoma Metropolitana, y Doctor en Pedagogía por la Universidad Nacional Autónoma de México. Desarrolla las líneas de investigación: Investigación de la investigación educación educativa, y Producción y fragmentación de subjetividades, ambas desde la perspectiva de la Economía de las prácticas sociales de Pierre Bourdieu. Actualmente es profesor-investigador de tiempo completo en el Área Académica de Ciencias de la Educación de la Universidad Autónoma del Estado de Hidalgo, México. ORCID ID: 0000-0003-2440-5494. Email: aceron@uaeh.edu.mx

\section{Eva Ma Galán Mireles}

Es Licenciada en Comunicación y Periodismo por la Universidad Nacional Autónoma de México, Maestra en Tecnología Educativa por la Universidad Autónoma del Estado de Hidalgo. Ha desempeñado diversas funciones en las áreas profesionales y académicas además como Evaluadora Analista de los Comités Interinstitucionales de Evaluación de la Educación Superior (CIEES) México. Desarrolla la línea de investigación: Usos sociales de las Tecnologías de la Información y la Comunicación. Actualmente es profesora de asignaturas en diversas universidades de México en la modalidad presencial y virtual. ORCID ID: 0000-0002-6470-5216. Email: eva.galan.mireles@gmail.com 